hepth@xxx/9207027

LPTENS-92/20

ENSLAPP-L-393/92

July 1992

\title{
SUPERSYMMETRIC BLACK HOLES FROM TODA THEORIES由
}

\author{
François DELDUC \\ Laboratoire de Physique Théorique de l'ENS Lyon, \\ 46 Allée d'Italie, 69364 Lyon CÉDEX 07, France. \\ Jean-Loup GERVAIS \\ Laboratoire de Physique Théorique de l'École Normale Supérieuref, \\ 24 rue Lhomond, 75231 Paris CÉDEX 05, France. \\ and \\ Mikhail V. SAVELIEV \\ Laboratoire de Physique Théorique de l'ENS Lyon, \\ 46 Allée d'Italie, 69364 Lyon CÉDEX 07, France.
}

\begin{abstract}
By the example of nonabelian Toda type theory associated with the Lie superalgebra $\operatorname{osp}(2 \mid 4)$ we show that this integrable dynamical system is relevant to a black hole background metric in the corresponding target space. In the even sector the model under consideration reduces to the exactly solvable conformal theory (nonabelian $B_{2}$ Toda system) in the presence of a black hole recently proposed in [1].
\end{abstract}

\footnotetext{
${ }^{1} \mathrm{LAT}_{\mathrm{E}} \mathrm{X}$ file available from hepth@xxx.lanl.gov (\# 9207027)

${ }^{2}$ Unité Propre du Centre National de la Recherche Scientifique, associée à l'École Normale Supérieure et à l'Université de Paris-Sud.

${ }^{3}$ On leave of absence from the Institute for High Energy Physics, 142284, Protvino, Moscow region, Russia.
} 
1. It was shown in paper [1] that exactly solvable nonabelian Toda systems [2] are relevant to two dimensional conformal field theories in the presence of a black hole. Here the tachyon fields arising as the potential terms in the corresponding Lagrangian, play the role of the $2 \mathrm{~d}$ cosmological terms. Moreover, the corresponding $B_{n}$-WZNW model is gauged by an appropriate nilpotent group, in distinction with those of Witten's scheme, see [3] and references therein. The simplest nontrivial example containing all main features characteristic of the black holes in the framework of this approach, is based on the Lie algebra $B_{2}$ supplied with a noncanonical gradation.

In the present note we discuss a supersymmetric black hole by the example of $N=1$ supersymmetric extension of nonabelian Toda system associated with the classical finite dimensional Lie superalgebra $\operatorname{osp}(2 \mid 4)$. Here we use such a choice of its simple roots that in the corresponding Dynkin diagram the first simple root is odd (it corresponds to $s l(1 \mid 1)$ superalgebra) and other two simple roots are even. With an appropriate gradation, this superalgebra provides, in the even sector, the reduction of the system under consideration to the case of the Lie algebra $B_{2}$.

2. Consider the classical Lie superalgebra $\mathcal{G}$ with the defining relations, see e.g., 四,

$$
\left[h_{i}, h_{j}\right]=0, \quad\left[h_{i}, X_{ \pm j}\right]= \pm k_{j i} X_{ \pm j}, \quad\left[X_{+i}, X_{-j}\right]=\delta_{i j} h_{i},
$$

where $k$ is the Cartan matrix of $\mathcal{G} ; h_{i}$ and $X_{ \pm i}, 1 \leq i \leq \operatorname{rank} \mathcal{G}$, are its Cartan and Chevalley generators. Here the numerical indices of the elements of the superalgebra $\mathcal{G}$ correspond to its roots, in particular, subscripts of the generators denote the simple roots. For the case under consideration $k$ is defined in accordance with the above mentioned Dynkin scheme. Realize the gradation of the $\operatorname{ssp}(2 \mid 4)$ by the Cartan element $H=h_{2}+2 h_{3}$ of its $\operatorname{osp}(1 \mid 2)$ subsuperalgebra with the odd elements $Y_{ \pm}=X_{\mp 1} \mp X_{ \pm 123}$, and even elements (generators of the $s l(2, C)$ subalgebra of $\operatorname{osp}(1 \mid 2)): H=\left[Y_{+}, Y_{-}\right]_{+}, X_{ \pm}=Y_{ \pm}^{2}$. Then the subspaces $\mathcal{G}_{m}, m=0, \pm 1, \pm 2$, of $\operatorname{osp}(2 \mid 4)=\oplus_{m} \mathcal{G}_{m}$, are the following:

$$
\begin{aligned}
\mathcal{G}_{0} & =\left\{X_{ \pm 2}, h_{i}, 1 \leq i \leq 3\right\} ; \\
\mathcal{G}_{ \pm 1} & =\left\{X_{\mp 1}, X_{\mp 12}, X_{ \pm 123}, X_{ \pm 1223}\right\} \\
\mathcal{G}_{ \pm 2} & =\left\{X_{ \pm 3}, X_{ \pm 23}, X_{ \pm 223}\right\} .
\end{aligned}
$$

Start with the $N=1$ superextension [5 of the Toda systems (Abelian as well as their nonabelian versions) associated with finite dimensional classical Lie superalgebras $\mathcal{G}$,

$$
D_{+}\left(g^{-1} D_{-} g\right)=\left[Y_{-}, g^{-1} Y_{+} g\right]_{+},
$$

where $g$ is a regular element of the Grassmann span of the Lie group $G_{0}$ with Lie algebra $\mathcal{G}_{0}$, and depends on the coordinates of the $2 \mid 2$ superspace $\mathcal{C}_{2 \mid 2} ; D_{ \pm}$are the supersymmetric covariant derivatives in this superspace. Then, in the case under consideration, parametrize the function $g$ as

$$
g=\exp \left\{a^{+} X_{+2}\right\} \exp \left\{a^{-} X_{-2}\right\} \exp \left\{\sum_{i=1}^{3} a_{i} h_{i}\right\}
$$


with the unknown functions (superfields) depending on the points in $\mathcal{C}_{2 \mid 2}$. We come to the following system of equations:

$$
\begin{aligned}
& D_{+} D_{-} a_{1}=e^{-a_{2}}-e^{-a_{1}+a_{2}-a_{3}}\left(1+a^{+} a^{-}\right), \\
& D_{+}\left[D_{-} a_{2}+a^{-} D_{-} a^{+}\right]=e^{-a_{1}+a_{2}-a_{3}}\left(1+a^{+} a^{-}\right), \\
& D_{+} D_{-} a_{3}=2 e^{-a_{1}+a_{2}-a_{3}}\left(1+a^{+} a^{-}\right), \\
& D_{+}\left[e^{-a_{1}-2 a_{2}+a_{3}} D_{-} a^{+}\right]=-e^{-2 a_{1}-a_{2}} a^{+}, \\
& D_{+}\left[e^{a_{1}+2 a_{2}-a_{3}}\left(D_{-} a^{-}-\left(a^{-}\right)^{2} D_{-} a^{+}\right)\right]=-e^{a_{1}+a_{2}-a_{3}} a^{-} .
\end{aligned}
$$

Now, it is the very time to note that there is such an element $H_{0} \equiv h_{1}-h_{3}$ which commutes with $Y_{ \pm}$, and, consequently, with $X_{ \pm}$, while $h_{2}$ commutes only with $X_{ \pm}$, and just due to this reason the r.h.s. of (5) in our case contains only $h_{1}$ and $H$ but not $h_{2}$ (as it should be in the even case). Then, making use the gauge properties of our system, equations (7) can be transformed, in terms of the functions

and

$$
\rho \equiv-\operatorname{Arsh}\left(a^{+} a^{-}\right)^{1 / 2}-i \frac{\pi}{2} ; \quad \psi \equiv-\frac{a_{1}+a_{3}}{2}+i \frac{\pi}{2}
$$

$$
\begin{aligned}
D_{+} \omega= & -\frac{1}{2\left(1+a^{+} a^{-}\right)}\left[a^{-} D_{+} a^{+}-\left(1+2 a^{+} a^{-}\right) a^{+} D_{+} a^{-}\right. \\
& \left.-2 a^{+} a^{-}\left(1+a^{+} a^{-}\right) D_{+}\left(a_{1}+2 a_{2}-a_{3}\right)\right], \\
D_{-} \omega= & \frac{1}{2\left(1+a^{+} a^{-}\right)}\left[-a^{+} D_{-} a^{-}+\left(1+2 a^{+} a^{-}\right) a^{-} D_{-} a^{+}\right] ;
\end{aligned}
$$

to the form

$$
\begin{aligned}
& D_{+} D_{-} \rho=\operatorname{ch} \rho e^{\psi}-\frac{\operatorname{sh} \rho}{\operatorname{ch}^{3} \rho} D_{+} \omega D_{-} \omega, \\
& D_{+}\left(\operatorname{th}^{2} \rho D_{-} \omega\right)-D_{-}\left(\operatorname{th}^{2} \rho D_{+} \omega\right)=0, \\
& D_{+} D_{-} \psi=\operatorname{sh} \rho e^{\psi} .
\end{aligned}
$$

The Lagrangian density $\mathcal{L}$ corresponding to the system (8) is the following:

$$
\mathcal{L}=\frac{1}{2} D_{+} \rho D_{-} \rho+\frac{1}{2} D_{+} \psi D_{-} \psi-\frac{1}{2} \operatorname{th}^{2} \rho D_{+} \omega D_{-} \omega+\operatorname{sh} \rho e^{\psi} .
$$

Therefore, the target-space metric $G$,

$$
G_{i j}=\operatorname{diag}\left(1,1,-\operatorname{th}^{2} \rho\right),
$$

contains the hyperbolic-tangent-square function (superfield) which, in even case, is characteristic for the black hole in the spirit of Witten. The potential term in (9) plays the role of the cosmological term in $\mathcal{C}_{2 \mid 2}$.

We are not going to discuss here more details concerning the properties of supersymmetric black holes in the framework of the given approach; only note once more that they are described by exactly solvable system of equations. A relation with the corresponding gauged supersymmetric WZNW model is quite evident.

Acknowledgements. One of the authors (M.S.) would like to thank A. Izergin, A. Sevrin, and P. Sorba for useful discussions; and ENSLAPP in Lyon for kind hospitality. 


\section{References}

[1] J.-L.Gervais, M.V.Saveliev: Black holes from non-abelian Toda theories, Preprint LPTENS-92/07, ENSLAPP-L-370/92, 1992;

to appear in Phys. Lett. B.

[2] A.N.Leznov, M.V.Saveliev: Lett. Math. Phys. 6, 505 (1982);

Comm. Math. Phys. 89, 59 (1983).

[3] E.Witten: Phys. Rev. D 44, 314 (1991);

Two-dimensional string theory and black holes, - in "Topics in quantum gravity“, Univ.of Cincinnati, April 1992.

[4] V.G.Kac: Comm. Math. Phys. 53, 31 (1977), F. Delduc, E. Ragoucy and P. Sorba Comm. Math. Phys. 146403 (1992).

[5] M.V.Saveliev: Comm. Math. Phys. 95, 199 (1984);

A.N.Leznov, M.V.Saveliev: Sov. J. Theor. Math. Phys. 61, 150 (1984); D.A.Leites, M.V.Saveliev, V.V.Serganova: - in "Group Theoretical Methods in Physics" V.I.Manko, M.A.Markov, eds., VNU Sci. Press 1, 255 (1986). 